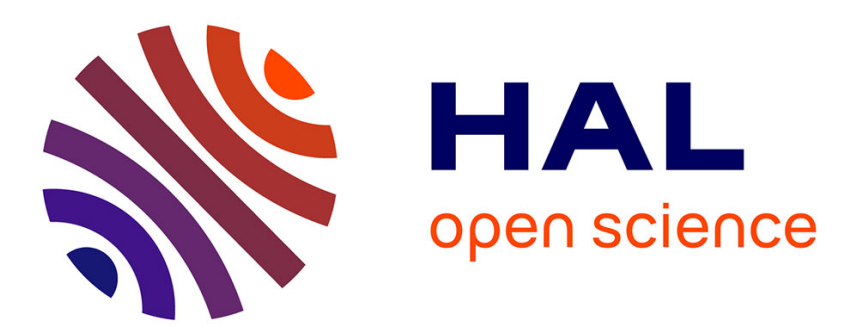

\title{
Improving the performance of low-gain designs for bounded control of linear systems
}

Frédéric Grognard, Rodolphe Sepulchre, Georges Bastin

\section{To cite this version:}

Frédéric Grognard, Rodolphe Sepulchre, Georges Bastin. Improving the performance of low-gain designs for bounded control of linear systems. Automatica, 2002, 38 (10), pp.1777-1782. 10.1016/S00051098(02)00086-9 . hal-01091545

\section{HAL Id: hal-01091545 \\ https://inria.hal.science/hal-01091545}

Submitted on 5 Dec 2014

HAL is a multi-disciplinary open access archive for the deposit and dissemination of scientific research documents, whether they are published or not. The documents may come from teaching and research institutions in France or abroad, or from public or private research centers.
L'archive ouverte pluridisciplinaire HAL, est destinée au dépôt et à la diffusion de documents scientifiques de niveau recherche, publiés ou non, émanant des établissements d'enseignement et de recherche français ou étrangers, des laboratoires publics ou privés. 


\title{
Improving the performance of low-gain designs for bounded control of linear systems
}

\author{
F. Grognard ${ }^{\dagger}$, R. Sepulchre ${ }^{\ddagger}$, G. Bastin $^{\dagger}$ \\ $\dagger$ Center for Systems Engineering and Applied Mechanics \\ Université catholique de Louvain, \\ Av. G. Lemaitre 4, B1348 Louvain-La-Neuve, Belgium. \\ Phone: +32-10-478030 Fax: +32-10-472180 \\ email:grognard@auto.ucl.ac.be \\ ‡ Institut Montefiore, B28 \\ Université de Liège, \\ B4000 Liège Sart-Tilman, Belgium. \\ email:r.sepulchre@ulg.ac.be
}

\section{Submitted to Automatica}

\section{Introduction}

The design of feedback control laws for linear systems subject to magnitude constraints on the control variable,

$$
\dot{x}=A x+B u \quad u \in \mathbb{R},|u| \leq 1
$$

has long been recognized as a significant nonlinear control problem. It is highly relevant in many applications. It has also stimulated many theoretical contributions. Notably, the time-optimal solution is a nice application of the maximum principle, which allows for analytical calculations of the switching surfaces, even though the complexity of the calculations is prohibitive except for low-dimensional systems $([1,10])$. More recently, special emphasis has been put on the design of stabilizing control laws that guarantee "large" regions of attraction $([7,8,3,5])$. Several of the proposed solutions rely on 
a one-parameter family of linear control laws $u=K(\epsilon) x$. As the parameter $\epsilon \rightarrow 0$, the norm $\|K(\epsilon)\|$ decreases (hence the name "low-gain" designs), so that the magnitude constraint $|u| \leq 1$ is satisfied in a large domain. At the same time, the guaranteed region of attraction of $x=0$ increases and may tend to the entire state space if $A$ has no eigenvalue with strictly positive real part.

In such designs, the tuning of the low-gain parameter $\epsilon$ involves two conflicting objectives. Large regions of attraction require small values of $\epsilon>0$ so that the input bound is never attained along the solutions. Obviously, this leads to cautious designs, resulting in slow convergence. In contrast, local performance would dictate a larger value of $\epsilon$, resulting in a reduced guaranteed region of attraction.

Based on the rationale that a small value $\epsilon_{0}$ is needed far from the origin and that a larger value $\epsilon_{f}$ is needed close to the origin, the present paper proposes an online adaptation of $\epsilon(t)$ aimed at the fastest possible evolution from $\epsilon_{0}$ to $\epsilon_{f}$ while guaranteeing closed-loop stability for the a priori selected set of initial conditions. Without loss of generality, $\epsilon_{f}$ can be normalized to one $\left(\epsilon_{f}=1\right)$.

Our design combines two ideas that have been recently discussed in the same context: the high-low gain philosophy of [6], which explicitely uses the infinite gain margin of Riccati based linear control laws $u=-B^{T} P(\epsilon) x$, and the gain scheduling proposed in [5], where $\epsilon(x)$ is chosen at every point so that $x$ lies on the boundary of the guaranteed region of attraction.

Our algorithm enforces invariance of the manifold $B^{T} P(\epsilon) x=0$ while enforcing the fastest possible increase of $\epsilon$. An analytical example and simulations suggest that this heuristic leads to accelerated convergence of the closed-loop solutions.

This paper is organized as follows. In Section 2, we expose methods to generate low-gain control laws. Existing methods to choose and schedule the gains are presented in Section 3. The algorithm is presented in Section 4, and it is analyzed and improved in Section 5. It is then illustrated on the double and triple integrator in Sections 6 and 7, with a comparison with the earlier scheme proposed by Megretski [5] and the time-optimal solution. Finally we give some conclusions.

\section{Low-gain designs}

In this paper, we restrict our attention to the family of saturated linear low-gain control laws in the form

$$
u=-\operatorname{sat}\left(B^{T} P(\epsilon) x\right)
$$


with

$$
\operatorname{sat}(y)=\operatorname{sign}(y) \min (|y|, 1)
$$

based on the quadratic Control Lyapunov Functions

$$
V(x, \epsilon)=x^{T} P(\epsilon) x
$$

The $\epsilon$-family of control laws (2.1) can be generated in various ways; one method uses the Riccati equation

$$
P(\epsilon) A+A^{T} P(\epsilon)-P(\epsilon) B B^{T} P(\epsilon)=-Q(\epsilon), \quad \epsilon \in(0, \infty)
$$

with $Q(\epsilon)>0$ (positive definite), continuous, such that $\lim _{\epsilon \rightarrow 0} Q(\epsilon)=0$ and $\frac{d Q(\epsilon)}{d \epsilon}>0([4,9])$.

If $(A, B)$ is asymptotically null controllable, that is $(A, B)$ is stabilizable and all the eigenvalues of $A$ are in the closed left half-plane, then $\lim _{\epsilon \rightarrow 0} P(\epsilon)=0, A-B B^{T} P(\epsilon)$ is Hurwitz for all $\epsilon>0$, and $\frac{d P(\epsilon)}{d \epsilon}>0$ $([2,11,12])$. In the remainder of the paper, null controllability of the considered linear system will always be assumed.

Megretski [5] suggests a simplified construction of $P(\epsilon)$ based on the Riccati inequality:

$$
P(\epsilon) A+A^{T} P(\epsilon)+P(\epsilon) W^{-2} P(\epsilon)-P(\epsilon) B B^{T} P(\epsilon)<0
$$

with $W>0$ a symmetric matrix. The advantage of (2.3) is that, if the region of attraction is only required to contain a given compact set $\Omega$, one does not need to explicitely solve the Riccati inequality for every $\epsilon$ : one can calculate a solution $P_{0}$ of $(2.3)$ such that $u=-$ sat $\left(B^{T} P_{0} x\right)$ ensures $\Omega$ to be contained in the region of attraction, as well as $P_{1}\left(>P_{0}\right)$ such that $u=-B^{T} P_{1} x$ ensures satisfying local performance. Then the matrix:

$$
P(\epsilon)=\left[(1-\epsilon) P_{0}^{-1}+\epsilon P_{1}^{-1}\right]^{-1} \quad \epsilon \in[0,1]
$$

is solution of (2.3), with $A-B B^{T} P(\epsilon)$ Hurwitz for all $\epsilon>0$, and $\frac{d P(\epsilon)}{d \epsilon}>0$. This construction does not guarantee global stabilization, but is useful for practical implementation, because it does not require the online solution of an Algebraic Riccati Equation. The resulting function $P(\epsilon)$ is an interpolation between $P_{0}$, which is chosen for stabilization, and $P_{1}$, which is chosen for performance. 


\section{Tuning of the low-gain parameter}

When $\lim _{\epsilon \rightarrow 0} P(\epsilon)=0$, the family of low-gain control laws (2.1) achieves semiglobal stabilization: given any compact set $\Omega$ to be included in the region of attraction, one can choose $\epsilon$ small enough such that $\left|B^{T} P(\epsilon) x\right|$ is bounded by 1 within a level set of $V(x, \epsilon)$ that includes $\Omega$. For a given initial condition $x_{0}$, the optimal $\epsilon$ is then:

$$
\epsilon\left(x_{0}\right)=\max \left\{\eta \in(0,1]: V(x, \eta) \leq V\left(x_{0}, \eta\right) \Rightarrow\left|B^{T} P(\eta) x\right| \leq 1\right\}
$$

With this choice, the compact set

$$
\mathcal{S}\left(\epsilon\left(x_{0}\right)\right)=\left\{x \in \mathbb{R}^{n} \mid V\left(x, \epsilon\left(x_{0}\right)\right) \leq V\left(x_{0}, \epsilon\left(x_{0}\right)\right)\right\}
$$

is guaranteed to be included in the region of attraction. For a fixed $\epsilon$, the maximal level set of the Lyapunov function within which $\left|B^{T} P(\epsilon) x\right| \leq 1$ is the minimal level set where the bound $\left|B^{T} P(\epsilon) x\right|=1$ is reached. Solving the optimization problem:

$$
\begin{array}{lr}
\min _{x \in \mathbb{R}^{n}} & x^{T} P(\epsilon) x \\
\text { s.t. } & B^{T} P(\epsilon) x=1
\end{array}
$$

(with the absolute value superfluous because of symmetry) yields:

$$
x_{\epsilon}=\frac{B}{B^{T} P(\epsilon) B}
$$

and $\left|B^{T} P(\epsilon) x\right| \leq 1$ within the set

$$
\mathcal{T}(\epsilon)=\left\{x \in \mathbb{R}^{n} \mid x^{T} P(\epsilon) x \leq \frac{1}{B^{T} P(\epsilon) B}\right\}
$$

Finding $\epsilon\left(x_{0}\right)$ then amounts to look for the largest $\epsilon$ such that $x_{0}$ lies within the set $\mathcal{T}(\epsilon)$, that is:

$$
\epsilon\left(x_{0}\right)=\max \left\{\eta \in(0,1]:\left(x_{0}^{T} P(\eta) x_{0}\right)\left(B^{T} P(\eta) B\right) \leq 1\right\}
$$

With the choices $(2.2)$ or (2.4), we have $\frac{d P(\epsilon)}{d \epsilon}>0$, and large regions of attraction $\Omega$ require small values of the parameter $\epsilon>0$. As a consequence, the resulting design is "cautious", that is, it uses little actuation near the origin and makes the convergence slow.

A first improvement of the above low-gain design is the "high-low" gain modification based on the observation that the control laws (2.1) have infinite gain margin. As a consequence, the region of attraction achieved with the 
control law $u=-\operatorname{sat}\left(k B^{T} P(\epsilon) x\right)$ still includes the set $\mathcal{S}\left(\epsilon\left(x_{0}\right)\right)$ with any gain $k \geq 1$. The limiting case for $k \rightarrow \infty$ results in the sliding mode control

$$
u=-\operatorname{sign}\left(B^{T} P(\epsilon) x\right)
$$

In this situation, full actuation is used throughout. However, the motion along the sliding surface $B^{T} P(\epsilon) x=0$ induces chattering and can be very slow when $\epsilon$ is small.

The idea that $\epsilon$ should be small far from the origin (for stability) and larger near the origin (for performance) suggests that the performance of low-gain designs will improve with an on-line adaptation of $\epsilon$. Megretski [5] proposes the choice

$$
\epsilon(x)=\max \left\{\eta \in(0,1]:\left(x^{T} P(\eta) x\right)\left(B^{T} P(\eta) B\right) \leq 1\right\}
$$

which corresponds to an online adaptation of the rule (3.5). It is shown in [5] that $V(x, \epsilon(x))$ then decreases along the solutions in the region of attraction, which is the entire state space if $P(\epsilon)$ satisfies (2.2), and which contains $\Omega$ if it satisfies (2.4).

If the initial condition is far from the origin, the parameter $\epsilon(x)$ will be initially small. However, it will increase as the solution approaches the origin. This gain scheduling can be stopped once $\epsilon$ has reached a value judged acceptable for local performance $(\epsilon=1)$.

The design flexibility of multiplying the control law by any gain $k \geq 1$ can

be combined with online adaptation of $\epsilon$, a gain $k$ proportional to $\frac{1}{\epsilon}$ being suggested by Lin (see e.g. [2]).

\section{Algorithm}

The online adaptation of $\epsilon$ presented in this paper is different from (3.8). It is based on the following observation: multiplying the low-gain control law by a large gain enforces the near-invariance of the subspace $\operatorname{ker} B^{T} P(\epsilon)$, at least in a neighborhood of the origin. In this region, rendering this subspace truly invariant will be less conservative and more relevant than ensuring $\dot{V}<0$ in the entire set $\mathcal{S}\left(\epsilon\left(x_{0}\right)\right)$. As a consequence, $\epsilon$ will be allowed to increase faster along the solutions. This suggests the following procedure in three phases:

- Phase 1: Reach the subspace $\operatorname{ker} B^{T} P(\epsilon)$ for some $\epsilon>0$ in a region such that the invariance condition $B^{T} P(\epsilon) x=0$ can be ensured for all future times with $|u| \leq 1$; 
- Phase 2: Increase $\epsilon$ as fast as possible while maintaining $B^{T} P(\epsilon) x=0$ and at the same time ensuring the control invariance of $B^{T} P(\epsilon) x=0$ for $\epsilon$ constant and $|u| \leq 1$;

- Phase 3: Once $\epsilon$ has reached the value 1 , for which the controller $u=-\operatorname{sat}\left(B^{T} P(1) x\right)$ ensures satisfying local performance, stop the adaptation and enforce the invariance condition $B^{T} P(1) x=0$, which guarantees convergence to the origin.

It is important to observe that, during Phase 2, the function $V(x, \epsilon)$ is no longer ensured to decrease along the solutions. This feature justifies the need for Phase 3. It also appears to be crucial for enabling a faster adaptation of $\epsilon$.

The following proposition characterizes the region of the state-space where Phase 2 is applicable. The considered region is characterized by $B^{T} P(\epsilon) x=$ 0 , and by the fact that the subspace $\operatorname{ker} B^{T} P(\epsilon)$, with $\epsilon>0$ fixed, can be made invariant for all future times with $|u| \leq 1$. This forward invariance condition then ensures convergence to the origin because $B^{T} P(\epsilon) x=0$ is a stable manifold.

Proposition 1 Let $\epsilon>0$, if the subspace $\operatorname{ker} B^{T} P(\epsilon)$ is not A-invariant, then it can be made control invariant with $|u| \leq 1$ within the region

$$
\left\{x \mid V(x, \epsilon)=x^{T} P(\epsilon) x \leq \bar{V}(\epsilon)\right\}
$$

with

$$
\bar{V}(\epsilon)=\frac{\left(B^{T} P(\epsilon) B\right)^{3}}{\left(B^{T} P(\epsilon) A P(\epsilon)^{-1} A^{T} P(\epsilon) B\right)\left(B^{T} P(\epsilon) B\right)-\left(B^{T} P(\epsilon) A B\right)^{2}}
$$

If $\operatorname{ker} B^{T} P(\epsilon)$ is A-invariant, then the entire subspace is kept invariant with $u=0$.

Proof Invariance of the subspace ker $B^{T} P(\epsilon)$ is achieved under the condition:

$$
\frac{d}{d t}\left(B^{T} P(\epsilon) x\right)=B^{T} P(\epsilon) A x+B^{T} P(\epsilon) B u=0
$$

This imposes the control

$$
u=-\frac{B^{T} P(\epsilon) A x}{B^{T} P(\epsilon) B}
$$

In order to satisfy the bound $|u| \leq 1$, we look for the maximum level $\bar{V}(\epsilon)$ of the Lyapunov function, such that, when $V(x, \epsilon) \leq \bar{V}(\epsilon)$ and $B^{T} P(\epsilon) x=0$, 
we have $\left|\frac{-B^{T} P(\epsilon) A x}{B^{T} P(\epsilon) B}\right| \leq 1$. This amounts to find the minimal level set where the bound $\left|\frac{-B^{T} P(\epsilon) A x}{B^{T} P(\epsilon) B}\right|=1$ is attained when $B^{T} P(\epsilon) x=0$, that is:

$$
\begin{array}{ll}
\min _{x \in \mathbb{R}^{n}} & x^{T} P(\epsilon) x \\
\text { s.t. } & B^{T} P(\epsilon) A x=B^{T} P(\epsilon) B \\
& B^{T} P(\epsilon) x=0
\end{array}
$$

Note that the absolute value in the first equality constraint is superfluous because of symmetry. Unless $\operatorname{ker} B^{T} P(\epsilon)$ is A-invariant, the Linear Quadratic problem (4.9) has a unique solution, given by

$$
\begin{aligned}
& x(\epsilon)=\frac{\left(B^{T} P(\epsilon) B\right)^{2} P(\epsilon)^{-1} A^{T} P(\epsilon) B-\left(B^{T} P(\epsilon) B\right)\left(B^{T} P(\epsilon) A B\right) B}{\left(B^{T} P(\epsilon) A B\right)^{2}-B^{T} P(\epsilon) B\left(B^{T} P(\epsilon) A P(\epsilon)^{-1} A^{T} P(\epsilon) B\right)} \\
& \bar{V}(\epsilon)=x(\epsilon)^{T} P(\epsilon) x(\epsilon) \\
& =\frac{\left(B^{T} P(\epsilon) A P(\epsilon)^{-1} A^{T} P(\epsilon) B\right)\left(B^{T} P(\epsilon) B\right)^{4}-\left(B^{T} P(\epsilon) A B\right)^{2}\left(B^{T} P(\epsilon) B\right)^{3}}{\left[\left(B^{T} P(\epsilon) A B\right)^{2}-\left(B^{T} P(\epsilon) A P(\epsilon)^{-1} A^{T} P(\epsilon) B\right)\left(B^{T} P(\epsilon) B\right)\right]^{2}} \\
& =\frac{\left(B^{T} P(\epsilon) B\right)^{3}}{\left(B^{T} P(\epsilon) A P(\epsilon)^{-1} A^{T} P(\epsilon) B\right)\left(B^{T} P(\epsilon) B\right)-\left(B^{T} P(\epsilon) A B\right)^{2}}
\end{aligned}
$$

To summarize, $B^{T} P(\epsilon) x=0$ can be made invariant with $|u| \leq 1$ when

$$
x^{T} P(\epsilon) x \leq \frac{\left(B^{T} P(\epsilon) B\right)^{3}}{\left(B^{T} P(\epsilon) A P(\epsilon)^{-1} A^{T} P(\epsilon) B\right)\left(B^{T} P(\epsilon) B\right)-\left(B^{T} P(\epsilon) A B\right)^{2}}
$$

A criterion to initialize Phase 2 of the algorithm is easily deduced from Proposition 1 . At any given point $x \in \mathbb{R}^{n}$, Phase 2 can be initialized provided there exist $\epsilon>0$ such that

$$
B^{T} P(\epsilon) x=0
$$

and

$$
\left(\left(B^{T} P A P^{-1} A^{T} P B\right)\left(B^{T} P B\right)-\left(B^{T} P A B\right)^{2}\right)\left(x^{T} P x\right) \leq\left(B^{T} P B\right)^{3}
$$

Note that (4.10) and (4.11) exactly characterize the two situations considered in Proposition 1.

We now give an explicit formulation of the algorithm. 


\section{Phase 1}

The first task of the control algorithm is to drive the solution to a region of the state-space where the conditions (4.10) and (4.11) are satisfied for some $\epsilon>0$.

A safe strategy to achieve this goal is to use the control law (3.7)

$$
u_{0}=-\operatorname{sign}\left(B^{T} P\left(\epsilon_{0}\right) x\right)
$$

with $P(\epsilon)$ taken from (2.2) or (2.4), where $\epsilon_{0}=\epsilon\left(x_{0}\right)$ is chosen according to (3.6). This control ensures convergence of the solution to the origin. The equation

$$
\frac{d}{d t}\left(B^{T} P\left(\epsilon\left(x_{0}\right)\right) x\right)=B^{T} P\left(\epsilon\left(x_{0}\right)\right) A x+\left(B^{T} P\left(\epsilon\left(x_{0}\right)\right) B\right) u_{0}
$$

then shows that the subspace $\operatorname{ker} B^{T} P\left(\epsilon\left(x_{0}\right)\right)$ is reached in finite time because $B^{T} P\left(\epsilon\left(x_{0}\right)\right) A x$ converges to zero. Moreover, inequality (4.11), with $\epsilon\left(x_{0}\right)$, is also satisfied in finite time because it is always satisfied in a neighborhood of the origin. This means that Conditions (4.10) and (4.11) will be satisfied in finite time, which terminates Phase 1.

\section{Phase 2}

The task of the controller during this phase of the algorithm is to increase $\epsilon$ up to 1 , the value desired for local performance, while keeping the invariance conditions (4.10) and (4.11) satisfied. This leads to two optimization problems, one or the other being used depending on whether constraint (4.11) is an equality or a strict inequality.

\section{Problem 1}

If (4.11) is a strict inequality, the constraint is inactive, and the task of the controller is then to maintain the invariance condition (4.10), that is

$$
\frac{d}{d t}\left(B^{T} P(\epsilon) x\right)=0
$$

that can be rewritten as:

$$
B^{T} P(\epsilon) A x+B^{T} P(\epsilon) B u+B^{T} \frac{\partial P(\epsilon)}{\partial \epsilon} x \dot{\epsilon}=0
$$

At singular points where $B^{T} \frac{\partial P(\epsilon)}{\partial \epsilon} x=0,(4.13)$ gives no constraint on $\dot{\epsilon}$, but $u$ must satisfy:

$$
u=\frac{-B^{T} P(\epsilon) A x}{B^{T} P(\epsilon) B}
$$


(In practice, we do not adapt $\epsilon$ at such points).

At regular points where $B^{T} \frac{\partial P(\epsilon)}{\partial \epsilon} x \neq 0$, we extract from (4.13) the adaptation rule

$$
\dot{\epsilon}=-\frac{B^{T} P(\epsilon) A x+B^{T} P(\epsilon) B u}{B^{T} \frac{\partial P(\epsilon)}{\partial \epsilon} x}
$$

and then solve the optimization problem

$$
\begin{aligned}
& \max \dot{\epsilon} \quad \text { s.t. } \\
& \frac{d}{d t}\left(B^{T} P(\epsilon) x\right)=0 \\
& |u| \leq 1
\end{aligned}
$$

The solution is

$$
u=-\operatorname{sign}\left(B^{T} \frac{\partial P(\epsilon)}{\partial \epsilon} x\right)
$$

Note that $\dot{\epsilon}>0$ because $\left|\frac{B^{T} P(\epsilon) A x}{B^{T} P(\epsilon) B}\right|<1$

\section{Problem 2}

If the constraint (4.11) is active, that is an equality constraint:

$$
\left(\left(B^{T} P A P^{-1} A^{T} P B\right)\left(B^{T} P B\right)-\left(B^{T} P A B\right)^{2}\right)\left(x^{T} P x\right)=\left(B^{T} P B\right)^{3}
$$

we impose that its time derivative be non positive, which yields an additional constraint on $\dot{\epsilon}$ :

$$
g(\epsilon)\left(x^{T} P(\epsilon) A x+x^{T} A^{T} P(\epsilon) x\right) \leq q(x, \epsilon) \dot{\epsilon}
$$

where

$$
\begin{aligned}
& g(\epsilon)=\left(B^{T} P(\epsilon) A P(\epsilon)^{-1} A^{T} P(\epsilon) B\right)\left(B^{T} P(\epsilon) B\right)-\left(B^{T} P(\epsilon) A B\right)^{2} \geq 0 \\
& q(x, \epsilon)=3\left(B^{T} P B\right)^{2}\left(B^{T} \frac{d P(\epsilon)}{d \epsilon} B\right)-\frac{d g(\epsilon)}{d \epsilon}\left(x^{T} P x\right)-g(\epsilon)\left(x^{T} \frac{d P(\epsilon)}{d \epsilon} x\right)
\end{aligned}
$$

with $q(x, \epsilon)$ not sign definite and $x^{T} P(\epsilon) A x+x^{T} A^{T} P(\epsilon) x<0$. The resulting optimization problem is: 


$$
\begin{aligned}
& \max \dot{\epsilon} \quad \text { s.t. } \\
& \begin{array}{l}
\frac{d}{d t}\left(B^{T} P(\epsilon) x\right)=0 \\
g(\epsilon)\left(x^{T} P(\epsilon) A x+x^{T} A^{T} P(\epsilon) x\right) \leq q(x, \epsilon) \dot{\epsilon} \\
|u| \leq 1
\end{array}
\end{aligned}
$$

If $q(x, \epsilon) \geq 0$, constraint (4.17) is inactive and the solution of Problem 2 is identical to that of Problem 1.

Else, the optimal $\dot{\epsilon}$ is

$$
\dot{\epsilon}=\min \left(\frac{g(\epsilon)\left(x^{T} P A x+x^{T} A^{T} P x\right)}{q(x, \epsilon)}, \max \left(\frac{B^{T} P A x-B^{T} P B}{B^{T} \frac{\partial P}{\partial \epsilon} x}, \frac{B^{T} P A x+B^{T} P B}{B^{T} \frac{\partial P}{\partial \epsilon} x}\right)\right)
$$

The solution trivially is $\dot{\epsilon}=\frac{g(\epsilon)\left(x^{T} P(\epsilon) A x+x^{T} A^{T} P(\epsilon) x\right)}{q(x, \epsilon)}$ when $B^{T} \frac{\partial P(\epsilon)}{\partial \epsilon} x=0$.

Using this expression of $\dot{\epsilon}$, the control $u$ is then obtained from (4.13).

\section{Phase 3}

Once $\epsilon$ has reached the desired value $\epsilon_{f}=1$, the adaptation is stopped and convergence to the origin is ensured with the control $u=-\frac{B^{T} P(1) A x}{B^{T} P(1) B}$ which ensures invariance of the stable manifold $B^{T} P(1) x=0$. For local performance, the control can be further switched to $u=-B^{T} P(1) x$ once the level set $x^{T} P(1) x=\frac{1}{B^{T} P(1) B}$ has been reached.

\section{Closed-loop behavior}

\subsection{Convergence}

Phase 1 is designed such as to terminate in finite time, while the objective of Phase 2 is to be terminated as fast as possible, with $\epsilon=1$. The normal evolution of the algorithm is thus that Phase 1 and Phase 2 terminate in finite time, and convergence of the solutions towards the origin is then ensured in Phase 3 (unless finite time convergence to the origin was already achieved at the end of Phase 1 or 2).

However, finite time termination of Phase 2 is not guaranteed. A situation can arise where $\epsilon \rightarrow \epsilon_{m} \leq 1$ as $t \rightarrow \infty$. In this case, Phase 2 never terminates, 
but convergence to the origin is ensured. Indeed, we differentiate the function $V(x, \epsilon)=x^{T} P(\epsilon) x:$

$$
\begin{aligned}
\frac{d}{d t} V(x, \epsilon) & =x^{T}\left(P(\epsilon) A+A^{T} P(\epsilon)\right) x+2 B^{T} P(\epsilon) x u+x^{T} \frac{\partial P(\epsilon)}{\partial \epsilon} x \dot{\epsilon} \\
& =x^{T}\left(P(\epsilon) A+A^{T} P(\epsilon)+2 P(\epsilon) B B^{T} P(\epsilon)\right) x+x^{T} \frac{\partial P(\epsilon)}{\partial \epsilon} x \dot{\epsilon} \\
& =x^{T}\left(P(\epsilon) A+A^{T} P(\epsilon)+2 P(\epsilon) B B^{T} P(\epsilon)+\frac{\partial P(\epsilon)}{\partial \epsilon} \dot{\epsilon}\right) x
\end{aligned}
$$

where the second equality holds because, during Phase 2, $x \in \operatorname{ker} B^{T} P(\epsilon)$. We know that $P(\epsilon) A+A^{T} P(\epsilon)+2 P(\epsilon) B B^{T} P(\epsilon)<0$ and that $\dot{\epsilon} \rightarrow 0$; therefore, there exists a time $t_{L}$ after which:

$$
P(\epsilon) A+A^{T} P(\epsilon)+2 P(\epsilon) B B^{T} P(\epsilon)+\frac{\partial P(\epsilon)}{\partial \epsilon} \dot{\epsilon}<0
$$

and $\dot{V}<0$, which implies convergence of the solution towards the origin.

Note that, when Phase 2 dos not terminate in finite time, the optimization problem 1 eventually becomes the only one that is used; indeed, $x^{T} P(\epsilon) x \rightarrow 0$ as $\epsilon \rightarrow \epsilon_{m}$, which means that constraint (4.11) will never be active when $x$ approaches the origin. The control law is then $u=$ $-\operatorname{sign}\left(B^{T} \frac{\partial P(\epsilon)}{\partial \epsilon} x\right)$ or $u=\frac{-B^{T} P(\epsilon) A x}{B^{T} P(\epsilon) B}(\leq 1)$, whether $B^{T} \frac{\partial P(\epsilon)}{\partial \epsilon} x \neq 0$ or not.

\subsection{Improvement of Phase 1}

Phase 1 is designed to terminate in finite time, that is when $B^{T} P\left(\epsilon\left(x_{0}\right)\right) x=$ 0 and the constraint (4.11) are satisfied. In fact, verifying the invariance conditions (4.10) and (4.11) for $\epsilon=\epsilon\left(x_{0}\right)$ is conservative because (4.10) and (4.11) could be satisfied for some larger value $\eta$ before they become satisfied for $\epsilon\left(x_{0}\right)$, allowing to initialize Phase 2 sooner, with a larger value of $\epsilon$. The conservatism would be eliminated by solving the nonlinear equation

$$
B^{T} P(\eta) x=0
$$

for $\eta$ on line and by checking the invariance condition (4.11), but this solution is obviously numerically expensive. A practical recommendation is as follows: given the initial condition $x_{0}$, solve off-line the nonlinear equation (5.19). If a positive solution $\eta\left(x_{0}\right)$ exists, keep track of the solution of (5.19) during Phase 1 , by using the adaptation law:

$$
\frac{d B^{T} P(\eta) x}{d t}=0
$$

which gives:

$$
B^{T} P(\eta) A x-\left(B^{T} P(\eta) B\right) \operatorname{sign}\left(B^{T} P\left(\epsilon\left(x_{0}\right)\right) x\right)+B^{T} \frac{\partial P(\eta)}{\partial \eta} x \dot{\eta}=0(5
$$


Extracting $\dot{\eta}$ from (5.20), dictates the evolution of $\eta$. It is then sufficient to check online whether the additional constraint (4.11) becomes satisfied for $\eta$, at a given time, in which case Phase 2 of the algorithm can be initialized with $\epsilon=\eta$. The solution of (5.19) may not exist for $x=x_{0}$ or may cease to exist along the closed-loop solution $x(t)$, in which case $\eta(t)$ escapes to infinity in finite time. In such situations, one will adopt the conservative solution of checking (4.10) and (4.11) for $\epsilon=\epsilon\left(x_{0}\right)$.

\section{The double integrator}

The algorithm is now illustrated on the double integrator:

$$
\left\{\begin{array}{l}
\dot{x}_{1}=x_{2} \\
\dot{x}_{2}=u \quad|u| \leq 1
\end{array}\right.
$$

The simplicity of this second order system allows for analytical calculations and yields an interesting analogy with the time-optimal control solution. Solving the Riccati equation (2.2) with:

$$
Q(\epsilon)=\left(\begin{array}{cc}
\epsilon^{4} & 0 \\
0 & \epsilon^{2}
\end{array}\right)
$$

results in the Lyapunov matrix:

$$
P(\epsilon)=\left(\begin{array}{cc}
\sqrt{3} \epsilon^{3} & \epsilon^{2} \\
\epsilon^{2} & \sqrt{3} \epsilon
\end{array}\right)
$$

and the family of low-gain controls is then:

$$
u=-B^{T} P(\epsilon) x=-\epsilon^{2} x_{1}-\sqrt{3} \epsilon x_{2} \quad \epsilon>0
$$

which is a typical low-gain control for second order systems. We consider that the behavior of the closed-loop system is satisfying when $\epsilon=1$. The invariance conditions (4.10) and (4.11) give respectively:

$$
\begin{aligned}
& x_{2}+\frac{\epsilon x_{1}}{\sqrt{3}}=0 \quad \text { with } \epsilon>0 \\
& \frac{\epsilon\left|x_{2}\right|}{\sqrt{3}} \leq 1
\end{aligned}
$$

The latter being obtained from (4.11):

$$
x^{T} P(\epsilon) x=\sqrt{3} \epsilon^{3} x_{1}^{2}+2 \epsilon^{2} x_{1} x_{2}+\sqrt{3} \epsilon x_{2}^{2} \leq \frac{6 \sqrt{3}}{\epsilon}
$$


taking equality (6.21) into account, or simply by imposing that the control law maintaining (6.21) invariant be bounded by 1 (which is sufficient for second order systems).

For the first phase of the algorithm, we follow (4.12) and use

$$
u=-\operatorname{sign}\left(x_{2}+\frac{\epsilon\left(x_{0}\right) x_{1}}{\sqrt{3}}\right)
$$

with $\epsilon\left(x_{0}\right)$ chosen as in (3.6), that is:

$$
\epsilon\left(x_{0}\right)=\max \left\{\eta \in(0,1]: \sqrt{3} \eta^{3} x_{01}^{2}+2 \eta^{2} x_{01} x_{02}+\sqrt{3} \eta x_{02}^{2} \leq \frac{1}{\sqrt{3} \epsilon}\right\}
$$

If $\epsilon\left(x_{0}\right)=1$, the control $u=-B^{T} P(1) x$ is applied and the algorithm is not used. Otherwise, in order to apply the algorithm, we notice that the two conditions (6.21) and (6.22) are simultaneously satisfied for some $\epsilon>0$ in the sectors delimited by the axis $x_{2}=0$ (i.e. $\frac{\epsilon\left|x_{2}\right|}{\sqrt{3}}=0$ ) and the manifold $x_{1}+x_{2}\left|x_{2}\right|=0$ (i.e. $\frac{\epsilon\left|x_{2}\right|}{\sqrt{3}}=1$ ). Phase 1 of the algorithm will be implemented only for initial conditions outside this sector, which is shaded on Figure 1.

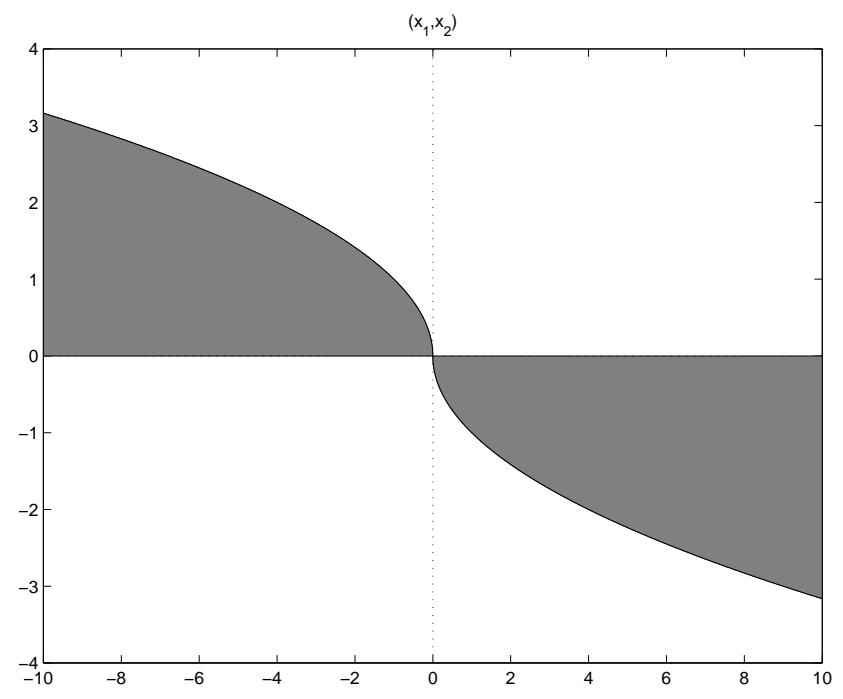

Figure 1: Sectors of initial conditions for which Phase 1 is not applied for the double integrator

Depending on the initial condition, Phase 1 terminates when the solution reaches the axis $x_{2}=0$ or the manifold $x_{1}+x_{2}\left|x_{2}\right|=0$.

During Phase 2, we use the maximum $\dot{\epsilon}$, while verifying

$$
\frac{d}{d t}\left(x_{2}+\frac{\epsilon x_{1}}{\sqrt{3}}\right)=0 \equiv \frac{x_{1} \dot{\epsilon}}{\sqrt{3}}+u+\frac{\epsilon x_{2}}{\sqrt{3}}
$$


and $\frac{\epsilon\left|x_{2}\right|}{\sqrt{3}} \leq 1$

The control algorithm is then:

$$
\left\{\begin{array}{l}
\dot{\epsilon}=-\frac{\sqrt{3} u+\epsilon x_{2}}{x_{1}} \\
u=-\operatorname{sign}\left(x_{1}\right)
\end{array}\right.
$$

until $\frac{\epsilon\left|x_{2}\right|}{\sqrt{3}}=1$, meaning that the manifold $x_{1}+x_{2}\left|x_{2}\right|=0$ is reached. On the manifold, the two constraints (4.10) and (4.11) give:

$$
\left\{\begin{array}{l}
\sqrt{3} u+\epsilon x_{2}+\dot{\epsilon} x_{1}=0 \\
\left(\epsilon u+\dot{\epsilon} x_{2}\right) x_{2} \leq 0
\end{array}\right.
$$

and the solution maximizing $\dot{\epsilon}$ is

$$
\left\{\begin{array}{l}
\dot{\epsilon}=\frac{\epsilon^{2}}{2 \sqrt{3}} \\
u=-\frac{1}{2} \operatorname{sign}\left(x_{2}\right)
\end{array}\right.
$$

The resulting control is thus the control $u= \pm \frac{1}{2}$ that keeps the manifold $x_{1}+x_{2}\left|x_{2}\right|=0$ invariant. The finite escape time observed for $\epsilon$ corresponds to finite time convergence of the solutions to the origin unless the adaptation is stopped at 1 in which case the final control (in Phase 3 ) is $u=-\frac{x_{2}}{\sqrt{3}}$, which achieves invariance of the manifold $x_{2}+\frac{x_{1}}{\sqrt{3}}=0$.

This control law is of course highly similar to the time-optimal solution. The control law is bang-bang and the switching surface is the one of the time-optimal control for a constraint $|u| \leq \frac{1}{2}$.

The value $\frac{1}{2}$ on the switching surface produces an average effect of the sliding mode control $u=-\operatorname{sign}\left(x_{1}+x_{2}\left|x_{2}\right|\right)$.

Our control algorithm and the control algorithm proposed in [5] can be compared based on the time taken by both schemes to reach the level set

$$
x^{T} P(1) x=\frac{1}{B^{T} P(1) B}
$$

that is:

$$
\sqrt{3} x_{1}^{2}+2 x_{1} x_{2}+\sqrt{3} x_{2}^{2}=\frac{1}{\sqrt{3}}
$$

Indeed, once the solutions are inside this set, their behaviors are very similar. The level set (6.24) is shown on Figure 2. Stars indicate the time instants or the points in state-space at which the solutions reach this level set. The faster convergence of the present algorithm can be explained by a faster adaptation of $\epsilon$ (see Figure 2): for a solution to reach the level set (6.24) from the initial condition $(10,0)$, the stars indicate that it takes 6.79 for our 
control algorithm versus 10.25 for the algorithm of [5], to be compared with 5.64 for the time optimal solution. This comes from the fact that, for a fixed $x$, the constraint $(3.8)$ on $\epsilon$, i.e.

$$
x^{T} P(\epsilon) x=\sqrt{3} \epsilon^{3} x_{1}^{2}+2 \epsilon^{2} x_{1} x_{2}+\sqrt{3} \epsilon x_{2}^{2} \leq \frac{1}{\sqrt{3} \epsilon},
$$

is tighter than (6.23). Comparing (3.8) and (4.11), the algorithm proposed in the present paper will accelerate the adaptation of $\epsilon$ whenever

$$
\frac{1}{\left(B^{T} P(\epsilon) B\right)}<\frac{\left(B^{T} P(\epsilon) B\right)^{3}}{\left(B^{T} P(\epsilon) A P(\epsilon)^{-1} A^{T} P(\epsilon) B\right)\left(B^{T} P(\epsilon) B\right)-\left(B^{T} P(\epsilon) A B\right)^{2}}
$$

for $\epsilon>0$.

\section{Simulations}

Figure 3 shows a simulation of the algorithm applied to the triple integrator:

$$
\left\{\begin{array}{l}
\dot{x}_{1}=x_{2} \\
\dot{x}_{2}=x_{3} \\
\dot{x}_{3}=u
\end{array} \quad|u| \leq 1\right.
$$

for the initial condition $(1,1,1)$. The family of low-gain control laws is generated by the Riccati equation (2.2), with the classical choice $Q(\epsilon)=\epsilon I$. An analytical solution cannot be found as easily as in the previous case, and the Riccati equation is therefore numerically solved online. Note that for higher dimensions, this calculation is expensive and justifies the use of (2.4), as suggested in [5].

Figure 3 shows a comparison of the algorithm presented in this paper with two other control schemes: the time optimal solution (which is still tractable for this example) and the online adaptation from [5] discussed in Section 3. The online adaptation is implemented with a large gain $\left(k=\frac{1}{\epsilon}\right)$ because it yields superior performance in this example. The difference between the three control schemes can best be seen in the evolution of $x_{1}$. The convergence of $x_{1}$ with our method is intermediate between the time-optimal solution and the solution [5]. As in the previous example, the upper hand of the present algorithm is also illustrated by the time that it takes for solutions to reach the level set

$$
x^{T} P(1) x=\frac{1}{B^{T} P(1) B}
$$



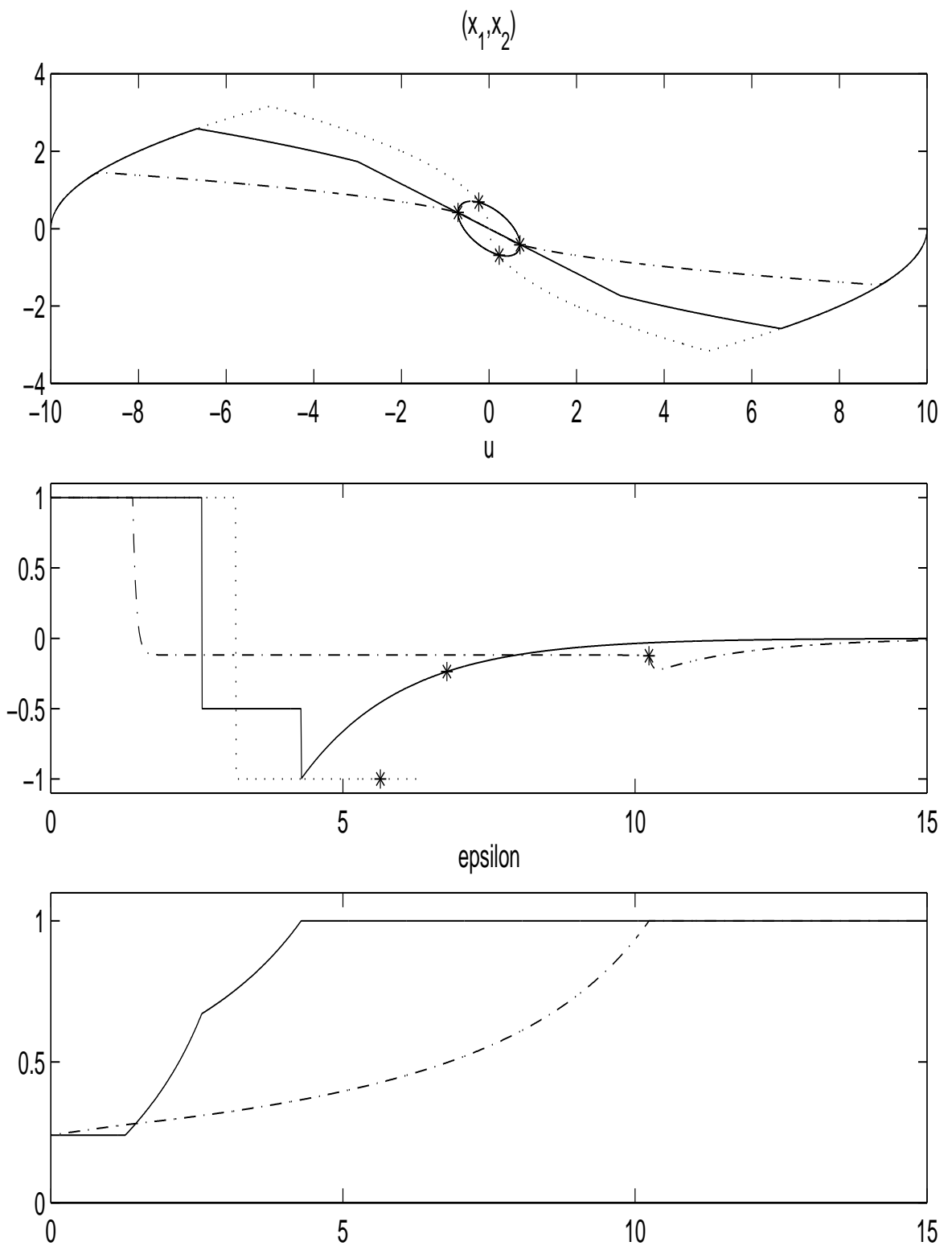

Figure 2: Control of the double integrator: time optimal method (dotted), Lin-Megretski's method with $k=\frac{1}{\epsilon}$ (dash-dotted) and our method (solid) $\left(\left(x_{10}, x_{20}\right)=(-10,0)\right)$ 
The faster adaptation of $\epsilon$, shown in Figure 3 allows for a faster convergence to this level set (7.56, to be compared with 9.83 for the algorithm of [5] and 6.58 for the time-optimal solution, for the initial condition $(1,1,1)$ ). This can be explained by inequality (6.25), which is again satisfied for all $\epsilon>0$. Finally, observe also that $V$ does not decrease during Phase 2.
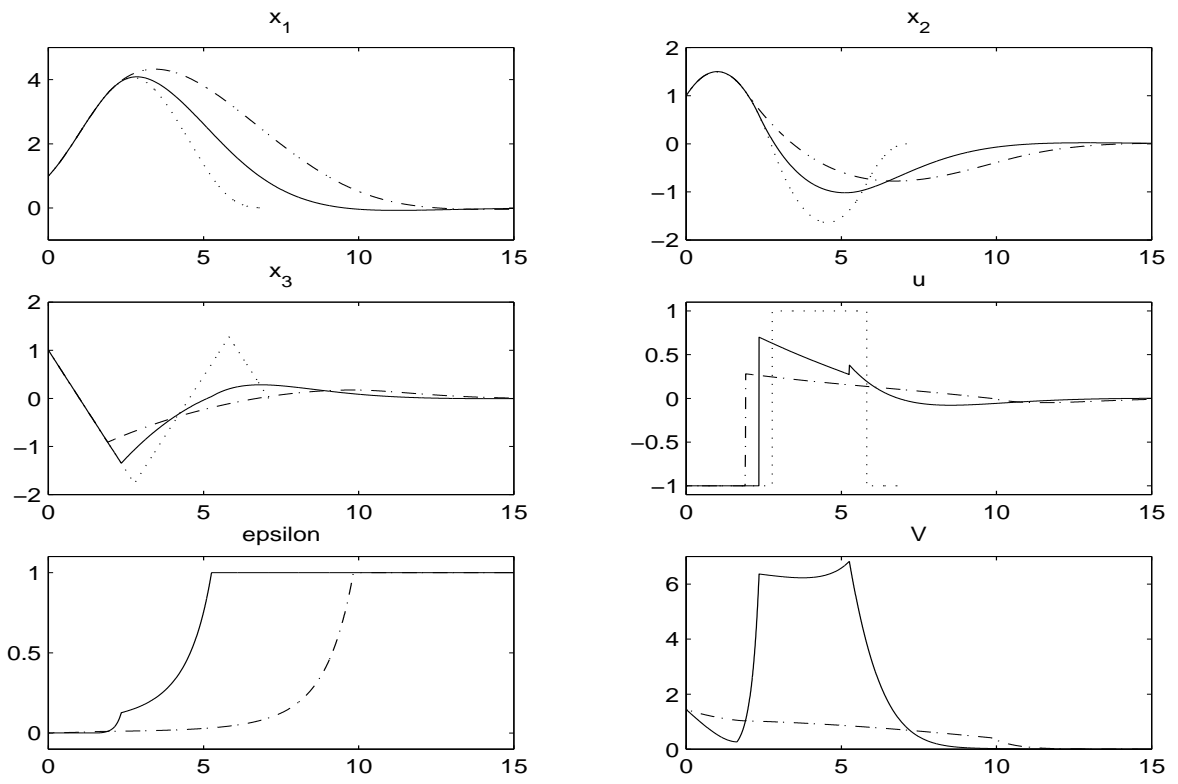

Figure 3: Control of the third order integrator: time optimal method (dotted), Lin-Megretski's method with $k=\frac{1}{\epsilon}$ (dash-dotted) and our method (solid) $\left(\left(x_{10}, x_{20}, x_{30}\right)=(1,1,1)\right)$

\section{Conclusion}

The control algorithm presented in this paper aims at improving the control performance of linear systems with bounded input. Starting from lowgain designs $u=-B^{T} P(\epsilon) x$ with infinite gain margin, we take advantage of the fact that, if a high gain is added and $u=-\operatorname{sat}\left(k B^{T} P(\epsilon) x\right)$, we have $B^{T} P(\epsilon) x \approx 0$ after a finite time. We explicitly use this observation in the adaptation rule $\dot{\epsilon}$, which leads to less conservative designs. Our design can be seen as a sliding mode design for which the sliding surface is calculated online. Further research in this direction will include the improvement of low-gain designs for general nonlinear feedforward systems. 


\section{References}

[1] E.B. Lee, L. Markus, Foundations of optimal control theory, Wiley \& Sons, 1967.

[2] Z. Lin, "Global Control of Linear Systems with Saturating Actuators", Automatica, vol. 34, no. 7, pp. 897-905, 1998.

[3] Z. Lin, A. Saberi, "Semiglobal exponential stabilization of linear systems subject to input saturation via linear feedbacks", Systems \& Control Letters, vol. 21, no. 3, pp. 225-239, 1993.

[4] Z. Lin, A.A. Stoorvogel, A. Saberi, "Output Regulation for Linear Systems Subject to Input Saturation", Automatica, vol 32, no. 1, pp. 29-47, 1996.

[5] A. Megretski, " $L_{2}$ BIBO output feedback stabilization with saturated control", Proceedings 13th IFAC World Congress, San Francisco, 1996, Vol D, pp. 435-440.

[6] A. Saberi, Z. Lin, A. Teel, "Control of Linear Systems with Saturating Actuators", IEEE Trans. on Automatic Control, vol. 41, no. 3, pp. 368$378,1996$.

[7] E.D. Sontag, H.J. Sussmann, "Nonlinear output feedback design for linear systems with saturating controls", Proceedings 29th Conference on Decision and Control, Honolulu, 1990, pp. 3414-3416.

[8] A. Teel, "Global stabilization and restricted tracking for multiple integrators with bounded controls", Systems \& Control Letters, vol. 18, pp. 165-171, 1992.

[9] A. Teel, "Semiglobal stabilizability of linear null controllable systems with input nonlinearities", IEEE Trans. on Automatic Control, vol. 40, no. 1, pp. 96-100, 1995

[10] T.L. Vincent, W.J. Grantham, "Nonlinear and optimal control", Wiley \& Sons, 1997.

[11] H.L. Trentelman, "Families of linear-quadratic problems: continuity properties", IEEE Trans. on Automatic Control, vol. 32, no. 4, pp. 323329, 1987. 
[12] J.C. Willems, "Least squares stationary optimal control and the algebraic Riccati equation", IEEE Trans. on Automatic Control, vol 16, no. 6, pp. 621-634, 1971. 